\title{
1 Early forest fire detection by vision-enabled wireless sensor networks
}

2 Suggested running head: Early fire detection by vision-enabled WSNs

3 Jorge Fernández-Berni ${ }^{A, C}$, Ricardo Carmona-Galán ${ }^{A}$, Juan F. Martínez-Carmona ${ }^{B}$, Ángel

$4 \quad$ Rodríguez-Vázquez ${ }^{A}$

$5{ }^{\text {A }}$ Institute of Microelectronics of Seville (IMSE CNM-CSIC-US), Avda. Américo Vespucio s/n,

$6 \quad$ Seville (Spain)

$7 \quad{ }^{B}$ Andalusian Forest Fire Suppression and Prevention Service (INFOCA), Avda. Manuel Siurot

850, Seville (Spain)

$9 \quad{ }^{\mathrm{C}}$ Corresponding author. Email: berni@imse-cnm.csic.es

10 Abstract: Wireless sensor networks constitute a powerful technology especially suitable for

11 environmental monitoring. With regard to wildfires, in particular, they enable low-cost fine-

12 grained surveillance of hazardous locations like wildland urban interfaces. This paper presents the work developed during the last four years targeting a vision-enabled wireless sensor network node for the reliable, early on-site detection of forest fires. The tasks carried out ranged from devising a robust vision algorithm for smoke detection to the design and physical implementation of a power-efficient smart imager tailored to the characteristics of such an

17 algorithm. By integrating this smart imager with a commercial wireless platform, we endowed the resulting system with vision capabilities and radio communication. Numerous tests were arranged in different natural scenarios in order progressively to tune all the parameters involved in the autonomous operation of this prototype node. The latest test carried out, involving the prescribed burning of a $95 \mathrm{~m} \times 20 \mathrm{~m}$ shrub plot, has confirmed the high degree of reliability of our approach in terms of both successful early detection and very low false alarm rate. 
1 Brief summary: This paper presents a vision-enabled wireless sensor network node for early

2 detection of forest fires. By integrating a prototype smart imager with a commercial wireless

3 platform, we endowed the resulting system with vision capabilities and radio communication.

4 The tests arranged have confirmed the high degree of reliability of our approach.

5 Additional keywords: smoke detection, false alarm rate, energy efficiency, artificial vision, 6 focal-plane processing, VLSI implementation

\section{$7 \quad$ Introduction}

8 A Wireless Sensor Network, WSN (Pottie 2000; Akyildiz et al. 2002), can be defined as a set of 9 low-power low-cost nodes with different sensing capabilities deployed throughout a region of interest. Processing and radio communication are also enabled on each sensor node. Due to these characteristics, WSNs are suitable for implementing environmental monitoring systems supported by fine-grained spatio-temporal sensing grids. With regard to forest fires, the WSNbased monitoring systems reported to date have been intended to predict rather than detect forest fires (Kremens et al. 2003; Chaczko and Amad 2005; Doolin and Sitar 2005; Son et al. 2006; Hefeeda 2007; Machado et al. 2010). This is because the available sensing modules have, in most cases, been restricted to scalar measurements like relative humidity, temperature or wind speed. These systems can therefore only determine the probability and eventual intensity of fire ignition from the observations of the environmental conditions provided by the sensors. Obviously, the nodes could still detect increases in temperature and decreases in barometric pressure and humidity from flame fronts before they burn, but very dense deployment is needed in order to detect a fire before it spreads (Fierens 2009).

A new possibility concerning WSN sensing capabilities has recently begun to be explored: that of multimedia sensing (Eren and Akan 2005; Akyildiz et al. 2007). In our case, the incorporation of artificial vision - meaning image sensing plus analog and digital processing plus video analytics and the obtaining of the targeted result - is of great interest. It would make it possible not only to monitor a certain area from the perspective of environmental conditions, 
1 but also to carry out visual inspection for the early detection of smoke or flames. However, the

2 implementation of vision hardware in WSN nodes is not at all a trivial matter. The visual

3 stimulus involves handling a massive flow of multidimensional information. Taking into

4 account the very strict power budget allocated to each node, the capture and digitization of one

5 image sequence alone could represent a significant percentage of its energy consumption. But

6 the critical point arises just afterwards. On one hand, the sequence could be simply transmitted

7 for remote processing, dramatically affecting the scalability and bandwidth of the network. On

8 the other hand, the node itself could deal with the image sequence using its own processing

9 capabilities. In this case, the nature of such processing is greatly influenced by energy constraints, and new strategies are therefore required which would allow early visual detection with the minimum possible power consumption. It is precisely on this need for new strategies to enable local image processing in WSN nodes that this paper focuses.

\section{Objectives}

14 Early detection and accurate location are two crucial points when it comes to preventing forest fires from spreading. The intervention of fire suppression resources becomes much more efficient if an alarm signal containing the geographical coordinates of the fire is delivered within a time interval of 15 minutes (INSA 2000). Ground systems (Sistema Bosque 1999; Kuhrt et al. 2001; Fire Watch 2004; Stipanicev et al. 2010) are currently the only systems capable of detecting a fire within a few minutes of its outbreak. They rely on cameras monitoring large areas. This means that a small number of cameras suffice to survey an extensive region. But it also means that detection is quite difficult as smoke can appear at very distant locations, triggering numerous false alarms (Arrue et al. 2000; Schroeder 2004) and requiring cameras featuring huge pixel resolution (Fernández-Berni et al. 2008). Moreover, these systems are quite expensive (Schroeder 2005). If infrared technology is to be used, costly calibration cycles push budgets up considerably. On the other hand, current WSN-based systems rely, as previously mentioned, on low-cost sensors which only monitor the environmental conditions around them. Very dense sensor deployment is therefore crucial to early detection. We propose to merge these 
two approaches together to achieve robust, scalable and reliable detection of forest fires with appropriate temporal and spatial resolution. The resulting system would be based on the sparse deployment of smart vision-enabled sensors constituting a wireless network. These sensors would on-site run a vision algorithm tailored to detect smoke arising from areas of vegetation which are small in comparison with the areas surveyed by cameras in ground systems. When a sensor detects smoke, a warning message is sent to a control center by multi-hopping through the network. A sketch of the system is shown in Fig.1, where $A_{S_{i}}$ represents the area surveyed by the sensor $S_{i}$. This system offers several advantages when compared to current automatic ground systems, namely:

- Robustness: The failure of one sensor affects only a very small fraction of the surveyed region.

- Scalability: Monitoring small areas, the layout of the sensor network can be adapted to the particular characteristics of the region to be surveyed.

- Reliability: The vision sensors analyze not landscape images but small areas of vegetation. Detection is therefore made easier and typical sources of false alarms like clouds or smoke rising from factories are avoided.

- Better temporal resolution: Thanks also to the small size of the areas surveyed by the sensors, smoke can be detected within short time intervals, typically a few minutes at most.

- Simpler smoke location: Complex GIS software is necessary for smoke location in commercial ground systems. In a system based on smart vision sensors, each sensor could store the geographic reference of the area it is surveying and include it in the alarm message. This would suffice easily to locate the fire. 
1

2

3 With respect to the WSN-based systems reported so far in literature, the incorporation of vision

Fig. 1: Sketch of the proposed system.

makes dense deployments for early detection unnecessary. The sensors do not now have to be

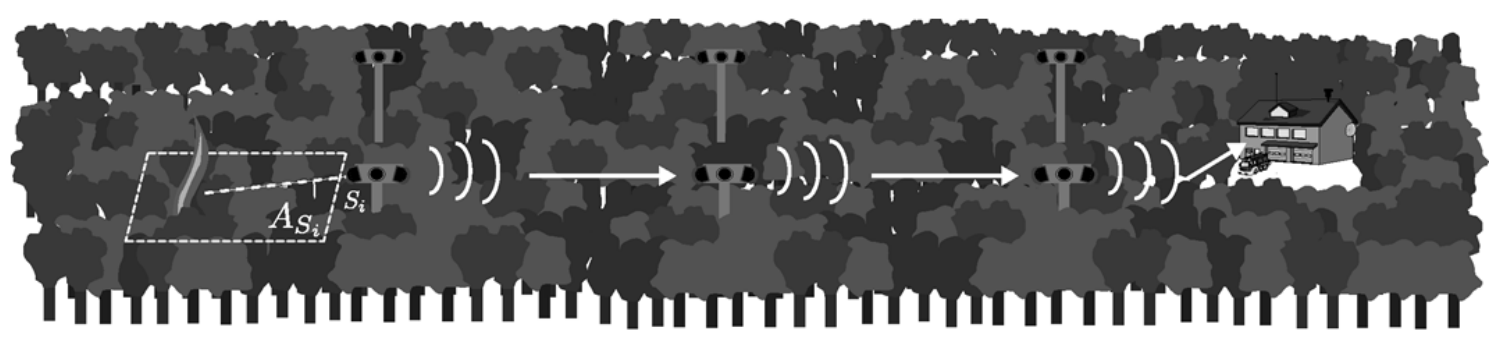
very close to the fire to detect its presence. This implies a significant reduction in the number of sensor nodes, and this in itself a significant step forward in terms of the cost and maintenance of the system as a whole.

Naturally, the benefits of the proposed system do not come totally free of charge. They essentially depend on the successful fulfilment of this work's primary objective; i.e. the efficient integration of vision capabilities into the sensing nodes. Bear in mind that we want the sensors constantly to run a vision algorithm at the minimum possible energy cost in order to prolong the life of their batteries as much as possible.

\section{Methodology}

\section{A vision algorithm for smoke detection}

A crucial element in our approach is a reliable vision algorithm for smoke detection in the scenario just described. Numerous vision algorithms for forest fire smoke detection have been reported in literature (de Vries and Kemp 1994; den Breejen et al. 1998; Vicente and Guillemant 2002; Gómez-Rodríguez et al. 2002; Toreyin et al. 2007; Krstinic et al. 2009). All of them analyze images coming from remote cameras surveying large areas. They have to deal with cloud motion, dust etc. in order to reduce the false alarm rate. In the system above proposed, however, the sensors watch small areas and most of the pixels of the images that are processed will therefore correspond to nearby vegetation. This means that the potential sources 
1 of false alarms are totally different. The movement which now has to be filtered is that of tree

2 leaves, birds or even people walking around. We would also like to point out that, from a more

3 general point of view, smoke detection can be seen as a case study in dynamic texture

4 recognition (Fernández-Berni et al. 2009).

5 As a preliminary step, the image plane is divided into regular regions with a size of $\mathrm{W} \times \mathrm{H}$ pixels.

6 The processing is then focused only on the mean value of the pixels corresponding to each

7 region. Despite its simplicity, we will demonstrate that such reduced scene representation

8 suffices for reliable smoke detection. Moreover, this objective can be achieved very efficiently

9 by using one of the processing primitives implemented by our smart imager, as described later.

10 In terms of image processing, the main effect caused by smoke rising against a vegetation

11 background is the increased luminance in the regions affected. Indeed, if RGB images are being

12 processed, the effect would be not only the increase of each component but also their equalization (Chen et al. 2006). Going one step further, we have found that the most sensitive component to the presence of smoke in such conditions is the blue component (Fernández-Berni et al. 2008). As an example, consider Fig. 2. We have marked a zone within a scene in which the background mainly comprises vegetation. The intensity histogram of the RGB components and the luminance is then represented under two situations: without smoke and with the presence of smoke. It can be seen that, without smoke, most of the pixels of the B component present the lowest intensity values. When smoke appears, the RGB components and the luminance increase and equalize their intensities. Note that the vertical axis of the histograms represents the number of pixels of the corresponding component featuring the intensity value indicated by the horizontal axis, within a scale of 256 values. 

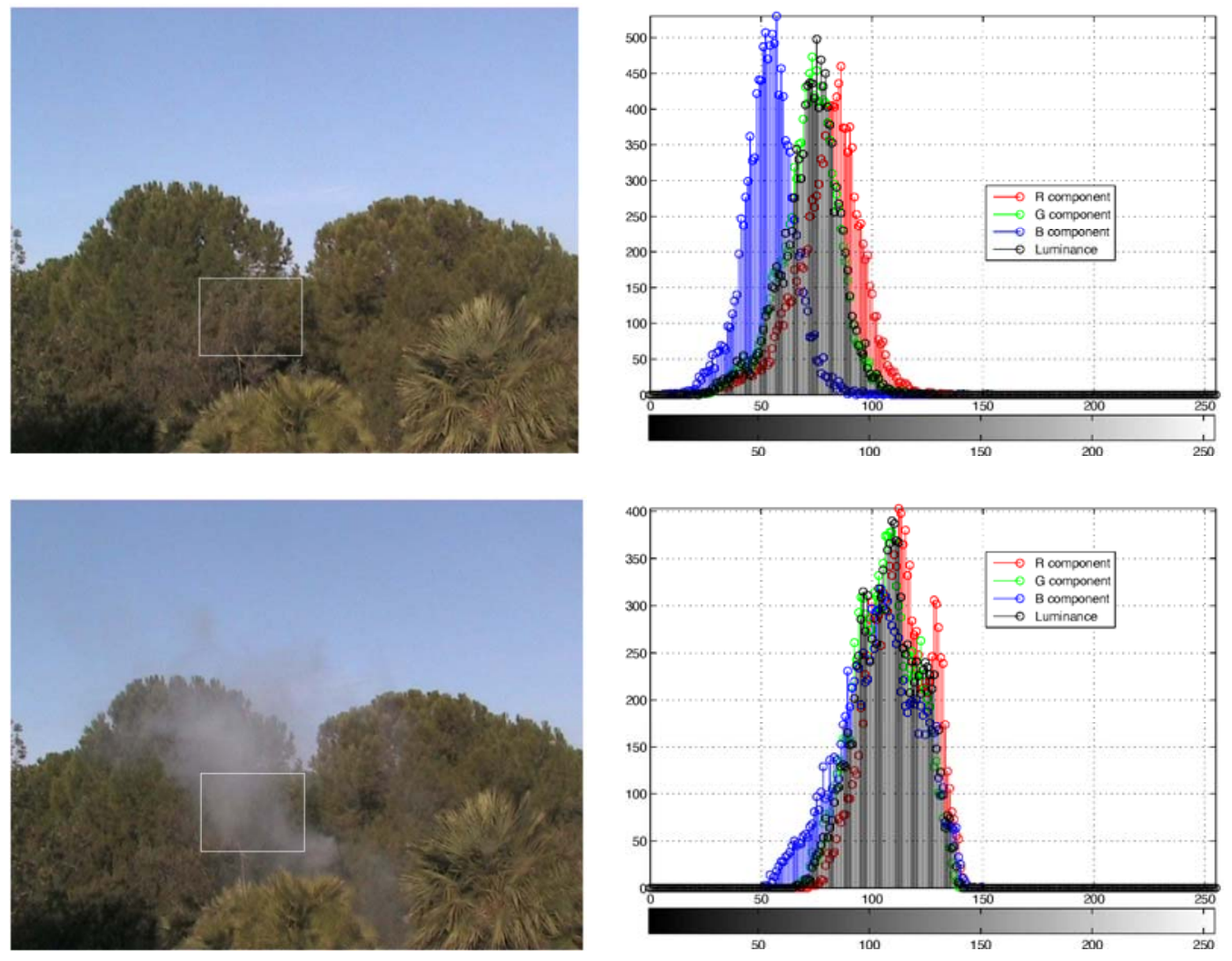

1 Fig. 2: Intensity histogram of the RGB components and luminance of a vegetation zone without smoke and with the presence of smoke.

3 To establish a numerical reference, we have calculated the normalized average increase, with

4 reference to the background, undergone by each component of the pixels within the marked 5 zones when smoke appears. The results are summarized in Table 1 . We can see that the 6 appearance of smoke among vegetation means a greater increase for the B component than for 7 the $\mathrm{R}$ and $\mathrm{G}$ components and the luminance. The $\mathrm{B}$ component is therefore the most sensitive to 8 the presence of smoke. These results are coherent with the reflectance spectra of typical fresh 9 and dry vegetation (Jacquemoud et al. 1994).

Table 1. Normalized average increase, with reference to the background, undergone by each component of the pixels within the zones marked in Fig. 2 when smoke appears.

\begin{tabular}{lcccc}
\hline & R component & G component & B component & Luminance \\
\hline Increase & $12.5 \%$ & $13.4 \%$ & $19.5 \%$ & $13.8 \%$ \\
\hline
\end{tabular}


1 All in all, the first stage of the algorithm has to do with a possible increase detected at any of the

$2 \mathrm{~W} \times \mathrm{H}$ regions in the image plane division. A region $(k, l)$ will be considered a candidate to contain smoke when its foreground intensity $I_{k l_{F}}$ fulfills the following equation:

$$
I_{k l_{F}} \geq I_{k l_{B}}+q
$$

4 where $I_{k l_{B}}$ is the background intensity of that same region $(k, l)$ and $q$ is a parameter indicating

5 the minimum increase in intensity a region must undergo if it is to be considered a candidate to

6 contain smoke. We are assuming that foreground images are captured at a fixed time interval

7 denoted as $T_{F}$. Note that Eq. (1) requires the existence of a background model. In our first

8 approach to this model (Fernández-Berni et al. 2010) we considered that, since the background

9 of the scenes inspected by the algorithm is mostly composed of vegetation, it rarely undergoes

10 significant changes. As long as no candidate region had been detected from the previous frame,

11 the background was therefore simply represented by sporadically updated frames. However, in the different field tests we carried out, we noticed that this very simple model, while working well under most operational conditions, was not adequate for vegetation areas affected by sudden changes in illumination; for example, when the sun is partially occluded by fast moving clouds. In such a case, a significant number of false candidate regions can be triggered, masking the presence of true candidate regions containing smoke. To deal with this, we modified the background model. Now, whenever certain spatio-temporal dynamics of candidate regions are dismissed, the background is updated with the next frame. Those changes which could prevent smoke from being adequately segmented and analyzed by the algorithm are therefore constantly being incorporated into the background representation. This modification has proven to endow the algorithm with very high reliability and robustness.

The second stage of the algorithm, once candidate regions have been detected, consists of searching for spatio-temporal patterns characteristic of smoke dynamics. This stage is divided into two phases: a detection phase and a confirmation phase. The detection phase starts when the first candidate regions are discovered, an instant denoted as $t_{0}$, and finishes at $t=t_{D}$. The confirmation phase is then initiated. This phase will last $T_{C}$ seconds at most if smoke is really 
1 present, finishing at time $t=t_{C}$ by sending an alarm message. The internal processing in both

2 phases works as follows. First of all, in order to consider that smoke is present at the scene, a

3 minimum number of candidate regions must exist. Let us define $N(t)$ as the number of

4 candidate regions at time instant $t$. This parameter can change every $T_{F}$ seconds - that is to say,

5 with each new foreground image captured. During the confirmation phase, the following 6 expression must be fulfilled:

$$
N(t) \geq N_{M I N} \quad\left\{t \in\left[t_{D}, t_{C}\right]\right\}
$$

7 where $N_{M I N}$ represents the minimum number of candidate regions for smoke to be considered.

8 Below that, changes are associated with a different source and the confirmation phase is 9 interrupted, returning to the pre-detection state.

10 Another important characteristic of smoke dynamics is their gradual appearance on the scene.

11 Once the first candidate regions are detected, new ones must gradually appear at least until

$12 N_{M I N}$ at $t=t_{D}$. This can be described by means of two conditions. The first one is:

$$
t_{D}-t_{0} \leq T_{D_{M A X}}
$$

where $T_{D_{M A X}}$ represents the maximum time interval within which smoke must appear once the first candidate regions are detected. The second condition is:

$$
N(t)-N\left(t-T_{F}\right) \leq \Delta_{M A X} \quad\left\{t \in\left[t_{0}, t_{C}\right]\right\}
$$

where $\Delta_{M A X}$ expresses the maximum permitted growth of candidate regions between two consecutive foreground images during the smoke dynamics. Non-fulfilment of Eq. (3) again cancels the detection and returns the system to the pre-detection state, because the source of the changes (e.g. fog) is considered too slow to behave like smoke. Failing to hold Eq. (4) means that the considered object is growing faster than smoke, and therefore comes from a different source (e.g. a bird flying past).

Finally, smoke does not appear as candidate regions scattered throughout the scene. On the contrary, it is formed by compact clusters. Let us define $Z(t)$ as the number of 8 -connected 
1 candidate region clusters. Just like $N(t), Z(t)$ can change with every foreground image. A

2 suitable compactness condition for smoke can be described as:

$$
Z(t) \leq Z_{M A X} \quad\left\{t \in\left[t_{0}, t_{C}\right]\right\}
$$

3 where $Z_{M A X}$ is the maximum permitted number of 8-connected candidate region clusters during

4 the smoke dynamics. In other words, failing to hold Eq. (5) means that a different source, for 5 example a flock of birds, is spreading changes in the scene.

6 As a summary, the flowchart of the algorithm is depicted in Fig. 3. Note that the objective is

7 that the different parameters defined work collaboratively in order to enable successful detection

8 within a set of conditions. Each parameter contributes to dismissing undesired dynamics by

9 encoding a certain characteristic of the spatio-temporal dynamics of a smoke plume. As a

10 whole, they ideally represent a number of features exclusively associated with such dynamics. 


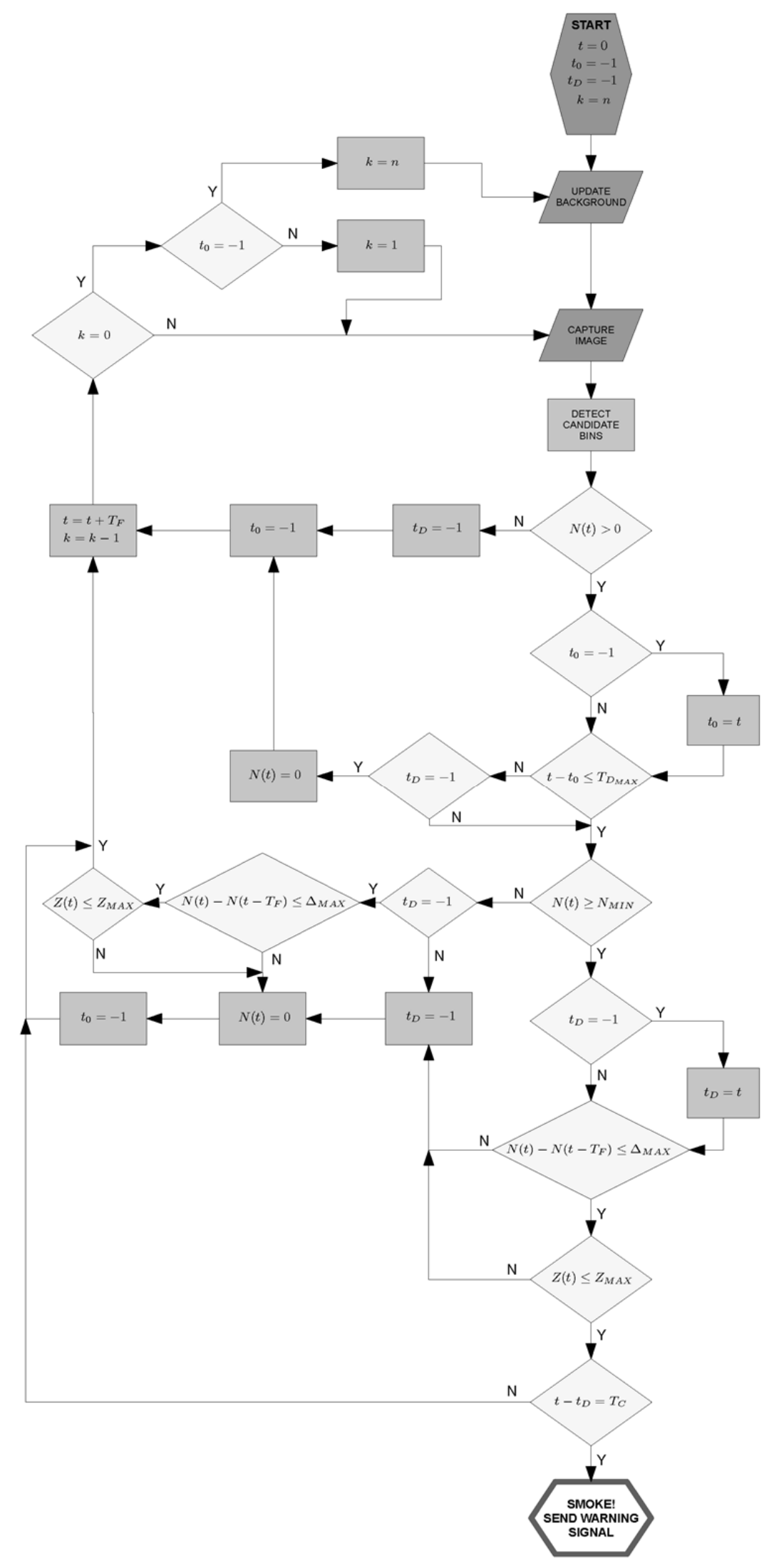

Fig. 3: Flowchart of the algorithm 
2 In order to set the numerical values of the different parameters of the algorithm and test the

3 algorithm itself, we recorded some sequences in a natural environment, a public park in Seville.

4 Approximately 80 minutes were recorded, with 16 sequences showing the gradual appearance

5 and natural evolution of smoke in scenes in which the background was basically vegetation.

6 Commercial pyrotechnic smoke generators were located at different distances from the three

7 camcorders used, ranging approximately from $20 \mathrm{~m}$ to $100 \mathrm{~m}$. To extract the parameters, 9 of the

816 sequences were analyzed. The rest were used to test the algorithm. Sequences without smoke

9 were also recorded to check the false alarm rate. All this material, in PAL format (720x576px, $25 \mathrm{fps})$, is available at http://www.imse-cnm.csic.es/vmote.

11 The most important parameter of the algorithm is $T_{F}$. Firstly, it must be correlated with the temporal scale of the smoke dynamics, and secondly, the higher its value, the lighter the processing load associated with the algorithm and consequently the lower the power consumption of its implementation. To set $T_{F}$, we implemented a very simple motion detector in which the first frame of every sequence analyzed was considered the background. Foreground motion was then determined at pixel level for subsequent frames. Those pixels changing more than a certain threshold would belong to the foreground. By empirically adjusting this threshold in such a way that most of the pixels representing smoke were segmented, we were able to obtain the approximate number of smoke pixels per frame. These are shown in Fig. 4 for the 9 sequences analyzed. This magnitude, highly dependent on the temporal dynamics of smoke, presents a very smooth variation. Indeed, most of the abrupt changes can be tracked by sampling the sequences every second. We thus concluded by setting $T_{F}=1 \mathrm{~s}$. The remaining parameters were adjusted taking into account this value of $T_{F}$, so that, from then on, the sequences would be sampled every second. 


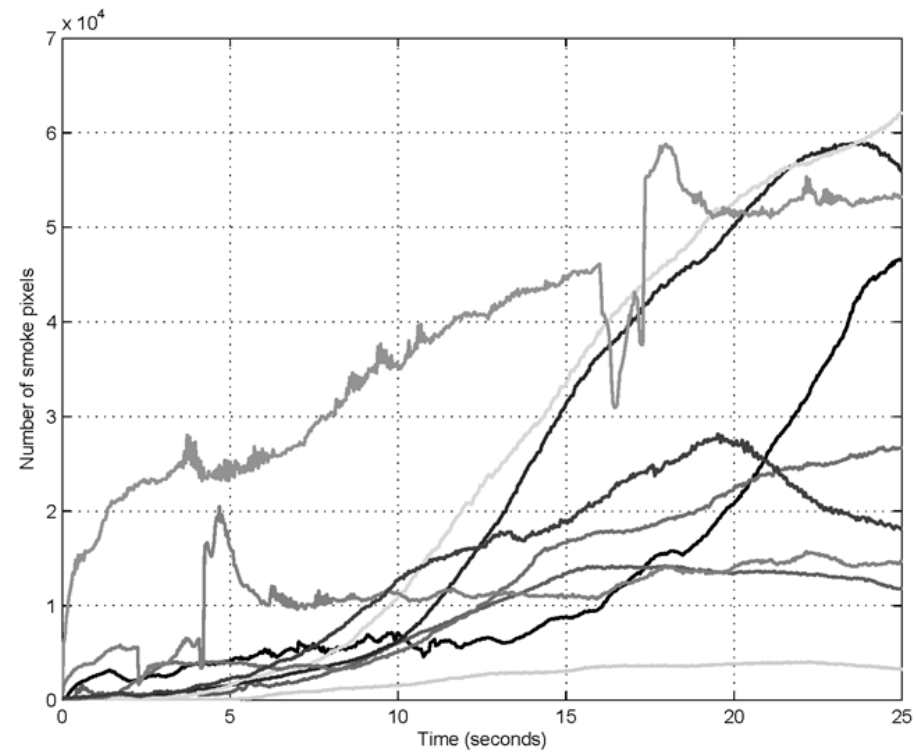

Fig. 4: Number of smoke pixels per frame for each of the 9 different sequences analyzed

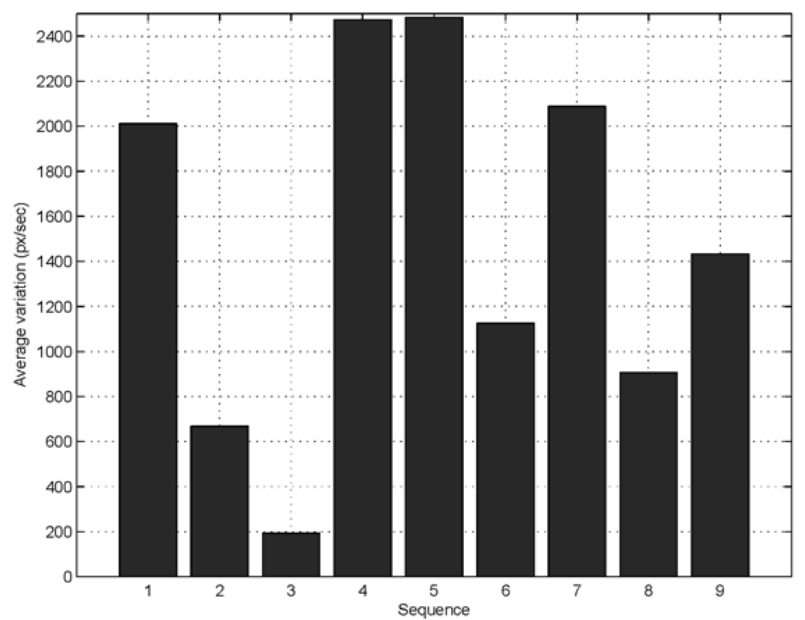

Fig. 5: Average variation in the number of pixels affected by smoke for each sequence. 
1 The next parameter considered is $q$. In this case, we simply computed the average increase,

2 normalized to the signal range, undergone by the values of the pixels affected by smoke. The

3 result is shown in Fig. 6. Accordingly, we set $q=10 \%$, where the percentage is referred to the

4 signal range. Note that this parameter may require further adjustment in order to accommodate

5 significant seasonal changes in the vegetation making up the background.

6

7

8 By applying Eq. (1) with the values of $\mathrm{W}, \mathrm{H}$ and $q$ just set, we were able to obtain the number

9 of candidate regions during the course of each sequence. The minimum value obtained was 17 .

Fig. 6: Normalized average increase in the pixels affected by smoke.

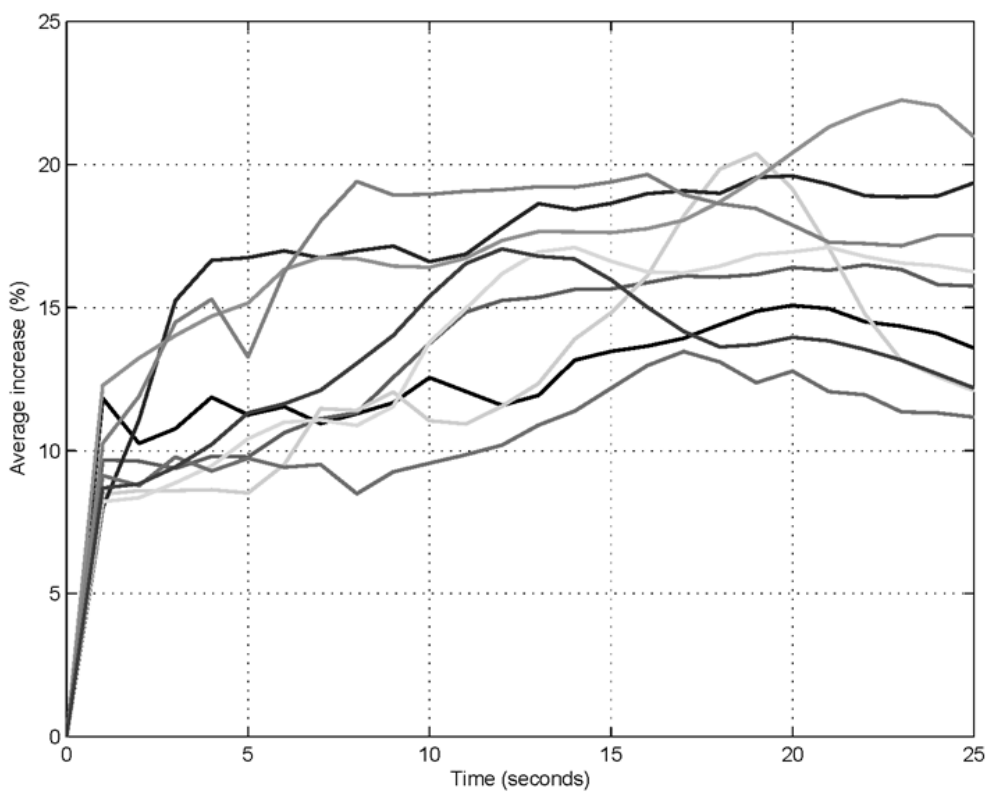

10 We set $N_{M I N}=14$ in order to allow a margin of three candidate regions. This choice implicitly

11 set $T_{D_{M A X}}=20 \mathrm{~s}$ and $T_{C}=4 \mathrm{~s}$. At this point, it was easy to adjust the value of $\Delta_{M A X}$ by computing the maximum growth rate of the candidate regions for each sequence. The greatest value found was 30 regions per second. We therefore set $\Delta_{M A X}=30$.

14 There is one parameter left: $Z_{M A X}$. To set it, the number of 8-connected candidate region clusters along every sequence was extracted. The maximum value, obtained only once in one of the sequences, was 6 . We therefore set $Z_{M A X}=6$, with no additional margin. 
1 Once the parameters were adjusted (see Table 2), we applied the algorithm to the 7 smoke

2 sequences that were not used for the setting process. Smoke was detected in all of them. The

3 algorithm was also applied to the sequences without smoke. No false alarm was triggered

4 despite the presence of different sources of motion like birds or tree leaves. Therefore, as far as

5 this reduced test bench is concerned, the algorithm achieved a reliability of $100 \%$. As an

6 example, in Fig. 7 we show the dynamics of each and every block making up the image plane

7 division for three sequences up until the time instant at which smoke was detected. Each region

8 is normalized with respect to its value in the background representation. Note that the time

9 instant at which smoke begins to appear in the scene can be easily distinguished due to the

10 progressive increase undergone by the regions affected.

Table 2. Summary of the algorithm settings.

12

\begin{tabular}{cc}
\hline Parameter & Value \\
\hline$T_{F}$ & $1 \mathrm{~s}$ \\
\hline $\mathrm{W} \times \mathrm{H}$ & $15 \times 12 \mathrm{px}$ \\
\hline$q$ & $10 \%$ \\
\hline$N_{M I N}$ & 14 \\
\hline$T_{D_{M A X}}$ & $20 \mathrm{~s}$ \\
\hline$T_{C}$ & $4 \mathrm{~s}$ \\
\hline$\Delta_{M A X}$ & 30 \\
\hline$Z_{M A X}$ & 6 \\
\hline
\end{tabular}

13
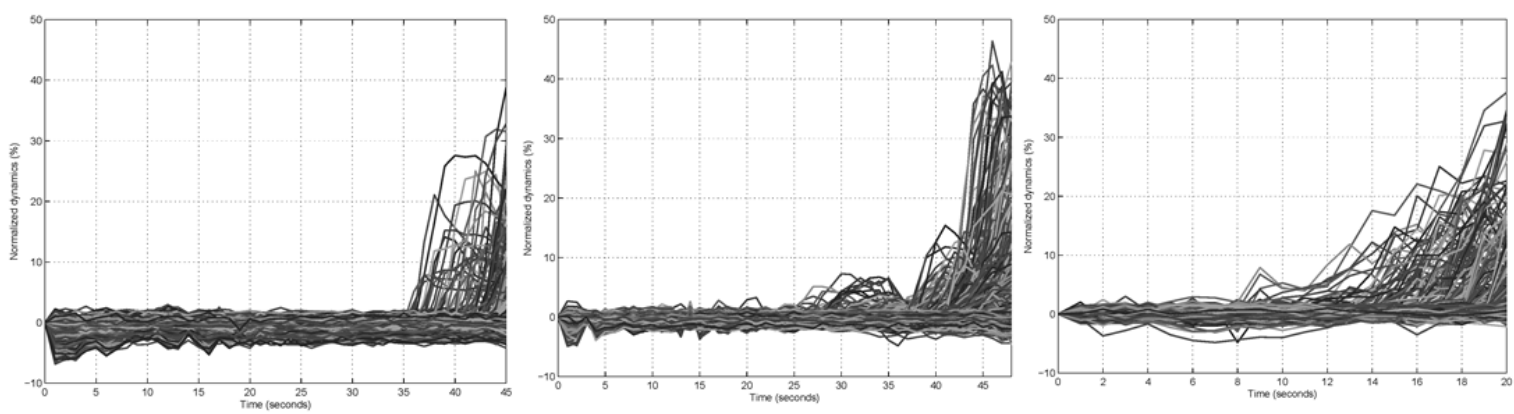

14 Fig. 7: Normalized dynamics (\%) of all the regions making up the image plane division for three recordings until smoke was detected. 


\section{$2 \quad$ Field tests with a commercial system}

3 The next step in our incremental approach to the problem, once the vision algorithm had been

4 tuned for smoke detection, was to programme it into a commercial autonomous vision system.

5 The objective was to carry out on-site surveillance while checking the algorithm in other

6 scenarios and dealing with potential operational problems. The system chosen was EyeRIS ${ }^{T M}$

7 v1.2, a general-purpose autonomous vision system built by AnaFocus Ltd.

8 (http://www.anafocus.com). The reasons for this choice were a) the availability of this system in

9 the laboratory when we had finished the preliminary tests on the algorithm and b) the

10 application development kit with which it is supplied and which enables the fast implementation

11 of standalone applications. The only modification to the algorithm for its implementation in

12 EyeRIS ${ }^{T M}$ was the image plane division. This modification is mandatory in order to keep the

13 other parameters unaltered. The elementary block was adjusted to the system's QCIF resolution

14 of $176 \times 144 \mathrm{px}$. Accurate adjustment would require elementary blocks of $3.66 \times 3 \mathrm{px}$, resulting from applying the reduction of resolution to the original size of these blocks, $15 \times 12 \mathrm{px}$. We finally decided to set blocks of $4 \times 4 \mathrm{px}$ for a slight increase in image simplification.

17 The algorithm was first tested in the laboratory. To this end, some of the video recordings containing smoke sequences were displayed on a computer screen on which EyeRIS ${ }^{T M}$ was focused. We also made sure that the algorithm's behaviour could be supervised in real time from a PC connected to the vision system. This real-time supervision resulted very useful in detecting minor problems during the field test carried out in the "Las Navas - El Berrocal" public forest in the province of Seville $(37.85 \mathrm{~N}, 6.04 \mathrm{~W})$. Two controlled burns of forest debris like those in Fig. 8 were overseen by personnel from the Andalusian Forest Fire Suppression and Prevention Service (INFOCA). The EyeRIS ${ }^{T M}$ system was placed on top of a three-meter high pole powered by a commercial $9 \mathrm{~V}$ battery connected to a DC-to-DC converter which supplied it with the adequate voltage. A camcorder was also placed on top of the pole in order to 
1 record the sequences from the same position as EyeRIS $S^{T M}$. The arrangement of both the

2 EyeRIS ${ }^{T M}$ system and the camcorder can be seen in Fig. 9. The pole was placed at a distance of 3 around 50 meters from the forest debris. Another camcorder was placed on a tripod at different

4 positions around the burns to record them from different perspectives. All the sequences can be

5 found and downloaded at http://www.imse-cnm.csic.es/vmote.

6

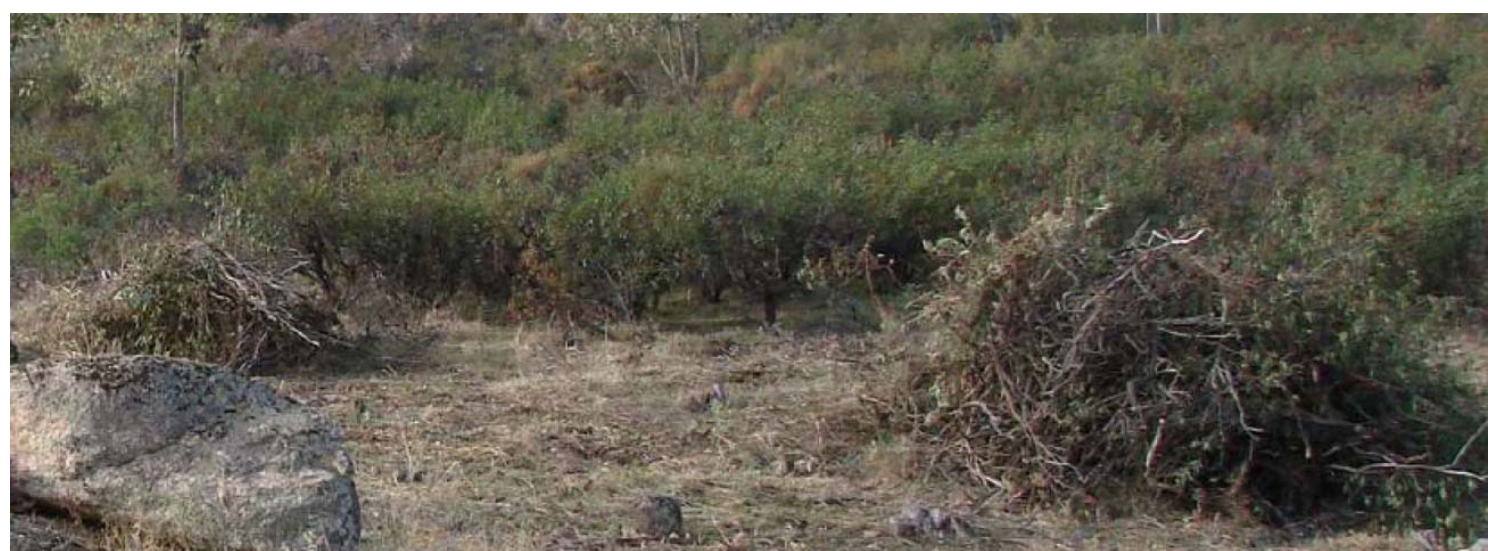

Fig. 8: Forest debris burnt during the field tests.
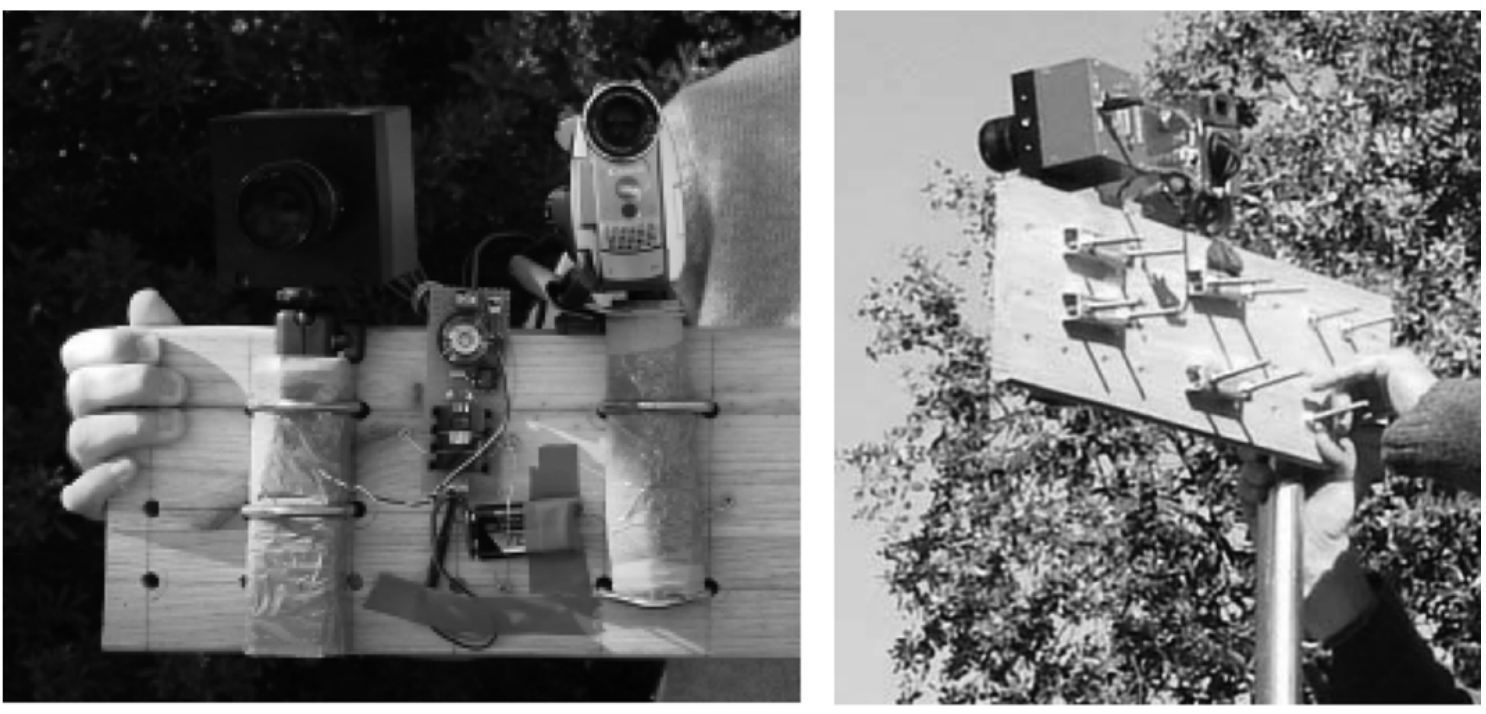

Fig. 9: Arrangement of the EyeRIS ${ }^{\mathrm{TM}}$ system (on the left in both photos) and the commercial camcorder (on the right in both photos) during the field tests. 
1 Smoke was detected without false alarms in both burns. In the first, the alarm was triggered at

$22 \mathrm{~min} 50 \mathrm{sec}$ from ignition whereas in the second the alarm was delivered after $57 \mathrm{sec}$. Some

3 consecutive frames captured by EyeRIS ${ }^{T M}$ during the first burn along with their corresponding

4 candidate regions are shown in Fig. 10. The evolution of $N(t)$ and $Z(t)$ is depicted in Fig. 11

5 for both burns. The most remarkable aspect about the results is the algorithm's ability to filter

6 motion other than smoke. In fact, it can be seen from the image sequences extracted that two

7 potential sources of false alarms like the movement of tree leaves due to wind and the

8 movement of people crossing the scene are mostly filtered. The alarms are therefore

9 undoubtedly triggered by the smoke arising from the burns.

10

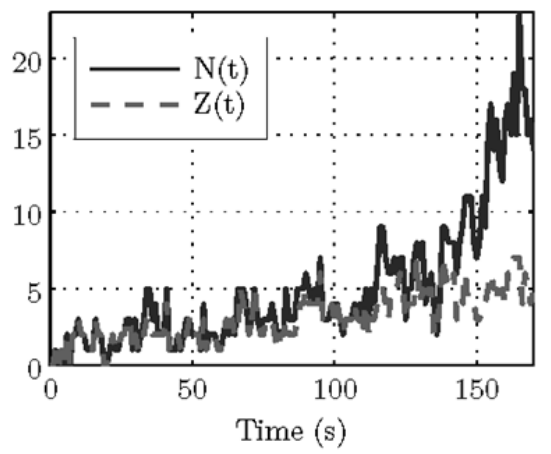

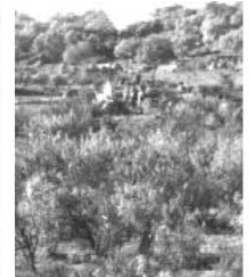
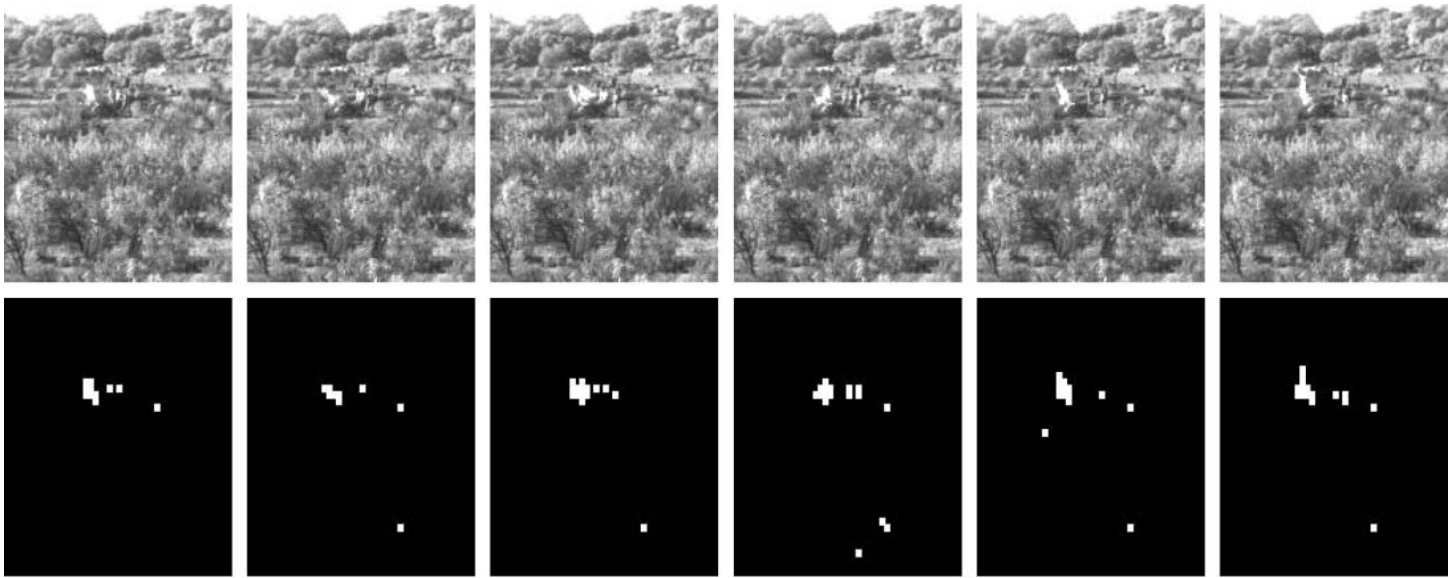

Fig. 10: Consecutive frames captured by EyeRIS ${ }^{T M}$ (first row) and their corresponding candidate bins (second row) during the first controlled burn.

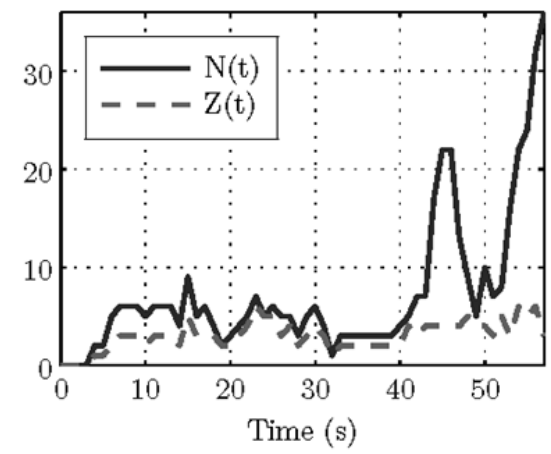

Fig. 11: Evolution of $N(t)$ and $Z(t)$ for the first and second burn respectively. 
2 Once the suitability of the algorithm for the early, on-site detection of forest fires was 3 confirmed, we finally addressed the implementation of a prototype vision-enabled WSN node.

4 A fundamental element of this node is the smart imager (Fernández-Berni et al. 2011-1). This 5 chip, called $F L I P-Q$, carries out image sensing, focal-plane processing running concurrently 6 with the sensing, and analog-to-digital conversion at very low energy cost $-5.6 \mathrm{~mW}$ in the worst

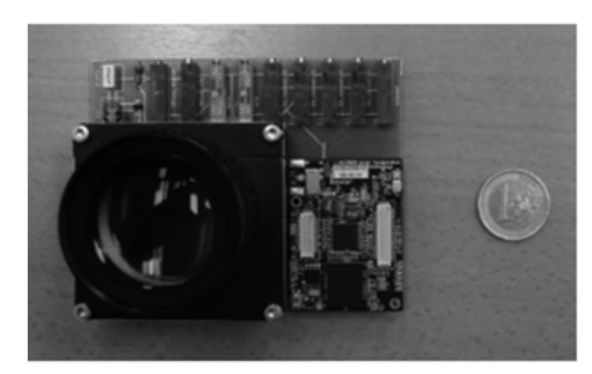
case. In this way, our imager can output from full-resolution digital images to different simplified representations of the scene which can then be reprogrammed in real time according to the results of the image processing. One of these simplified representations is the division of the image plane into regions of $\mathrm{W} \times \mathrm{H}$ pixels and the subsequent computation of their mean value, an operation required by the smoke detection algorithm. It is achieved in parallel for all the regions, no matter their size, the only energy cost being the power needed to capture a frame. This efficiency is crucial if the nodes are to have a long lifetime.

Wi-FLIP (Fernández-Berni et al. 2011-2) is the vision-enabled WSN node resulting from the integration of $F L I P-Q$ and Imote2, a commercial WSN platform from MEMSIC Corp. (www.memsic.com). This platform is built around a microprocessor which can operate in a low voltage low-frequency mode, hence allowing very low power operation. An 802.15.4-compliant radio is also integrated into the Imote2 system. Two snapshots of Wi-FLIP are shown in Fig. 12.

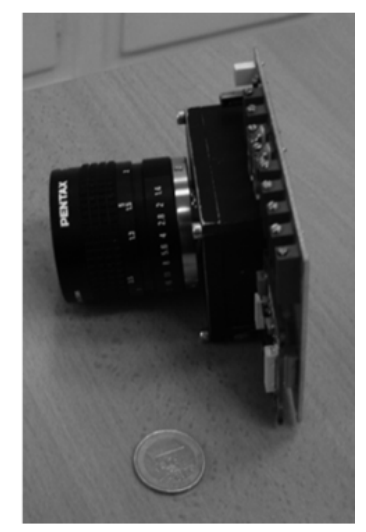

Fig. 12: $W i-F L I P$, a $10 \mathrm{~cm} \times 8 \mathrm{~cm}$ vision-enabled node for wireless applications. 


\section{Results and Discussion}

2 The smoke detection algorithm was programmed into Wi-FLIP. No additional modification was

3 necessary as the resolution of Wi-FLIP is the same as EyeRIS ${ }^{T M}$. Furthermore, since the

4 programming languages used in both systems are similar, the adaptation of the code written for

5 EyeRIS $^{T M}$ to $W i-F L I P$ was quite simple. To attain the prescribed frame rate for the algorithm,

$6 \quad 1 \mathrm{fps}$, the microprocessor had to be set to $416 \mathrm{MHz}$. This represented a power consumption of

7 around $155 \mathrm{~mA}$; that is, a node lifetime of around 10 hours for the three AAA batteries powering

8 the system. A small solar panel could be also used for continuous operation, but this option has

9 not yet been tested. Thanks to the availability of radio communication in Wi-FLIP, full-

10 resolution images are constantly sent via radio to a remote base station connected to a PC every

$1115 \mathrm{~s}$ after an alarm is triggered. This base station can be located within a range of $30 \mathrm{~m}$. In a real

12 deployment, the information transmitted by Wi-FLIP would be relayed by successive nodes

13 until it reached the corresponding base station.

14 As a first step, we arranged new tests in the same public park where the video sequences for the algorithm settings were recorded. Commercial pyrotechnics were again used as smoke generators The setting up of these tests was complicated by strong gusts of wind. Nevertheless, no false alarm was triggered during eight sequences of smoke generation and detection was successful in five of them. Smoke was not successfully detected in the others because, due to the wind, the pyrotechnic material burnt out before it had entered the field of view of Wi-FLIP sufficiently to be registered. In a real fire, smoke spreads steadily and should therefore eventually be detected. Some frames captured by a commercial camcorder and the corresponding smoke segmentation realized by Wi-FLIP are shown in Fig. 13. The last image corresponds to the first alarm image sent via radio. 

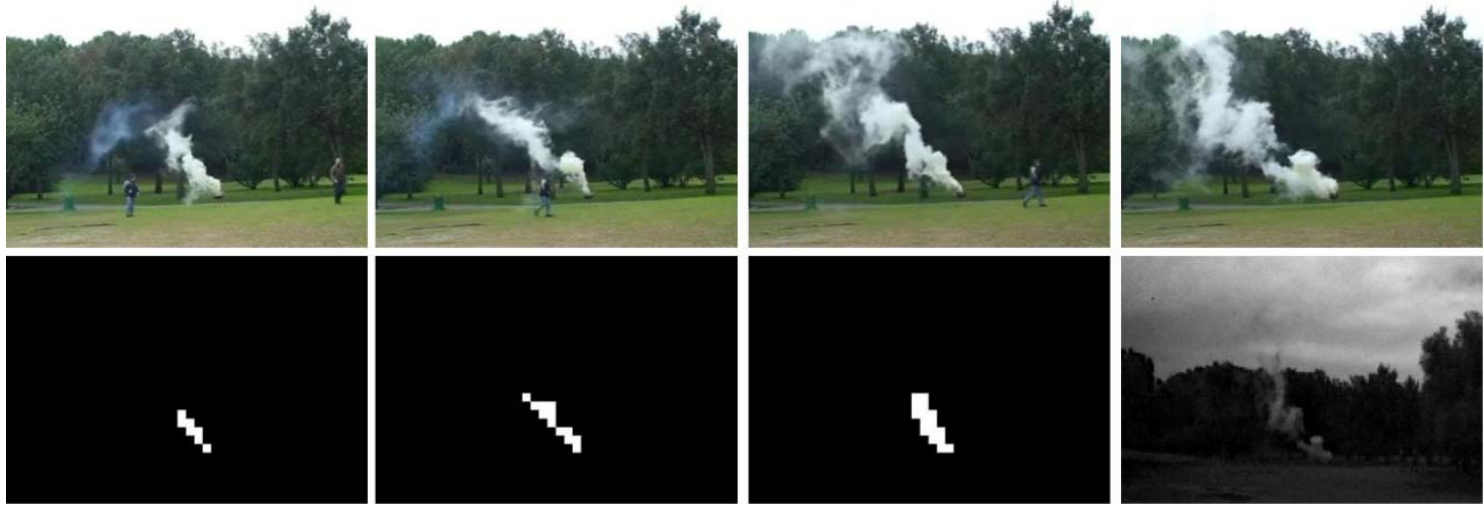

Fig. 13: Smoke segmentation and alarm image from Wi-FLIP.

The latest test consisted of the prescribed burning of a $95 \mathrm{~m} \times 20 \mathrm{~m}$ area of vegetation in the "Las

Navas - El Berrocal" public forest, once again in collaboration with the Andalusian Forest Fire Supression and Prevention Service. This area featured a smooth slope and different species of shrubs: Genista hirsuta, Lavandula stoechas and Cistus ladanifer. According to the Rothermel fire-spread model (Rothermel 1972) these constitute fuel model 6. Wi-FLIP was placed about $80 \mathrm{~m}$ away from the aforementioned area and monitored all the activity occurring in it for over two hours. A commercial camcorder also recorded a number of sequences. Two snapshots taken during the test are shown in Fig. 14. More material can be found at http://www.imsecnm.csic.es/vmote. With regard to the results obtained, it should first be mentioned that, prior to the burn, the area was mechanically divided into three zones of similar sizes according to the density of the vegetation. The first zone presented very sparse vegetation. This meant that very thin smoke was generated, and produced the only case in which Wi-FLIP did not trigger an alarm. In the second zone, the vegetation was denser and the smoke which appeared was therefore thicker. A first alarm was therefore triggered $5 \mathrm{~m} 28 \mathrm{sec}$ after ignition. With the fire still active, we reset $W i-F L I P$ so that the algorithm started to run again from zero. A new alarm was triggered $2 \mathrm{~m} 40 \mathrm{sec}$ after this reset operation. Finally, the third zone featured the densest vegetation. A first alarm was delivered $3 \mathrm{~m} 29 \mathrm{sec}$ after the fire was started there. We then repeated the $W i-F L I P$ reset operation performed for the second zone, and a second alarm was triggered $1 \mathrm{~m} 9 \mathrm{sec}$ afterwards. These alarms triggered even with the fire already spreading demonstrate the algorithm's capability to detect the presence of fire in a scene, regardless of its 
1 characteristics at any given moment. The first image sent via radio by Wi-FLIP for one of the

2 four alarms triggered is shown in Fig. 15. It is also especially remarkable that, despite the fact

3 that a great deal of people and vehicles were moving around, no false alarm was triggered either

4 before or after the prescribed burn. This is a key point concerning the reliability of our vision5 enabled WSN node.

6
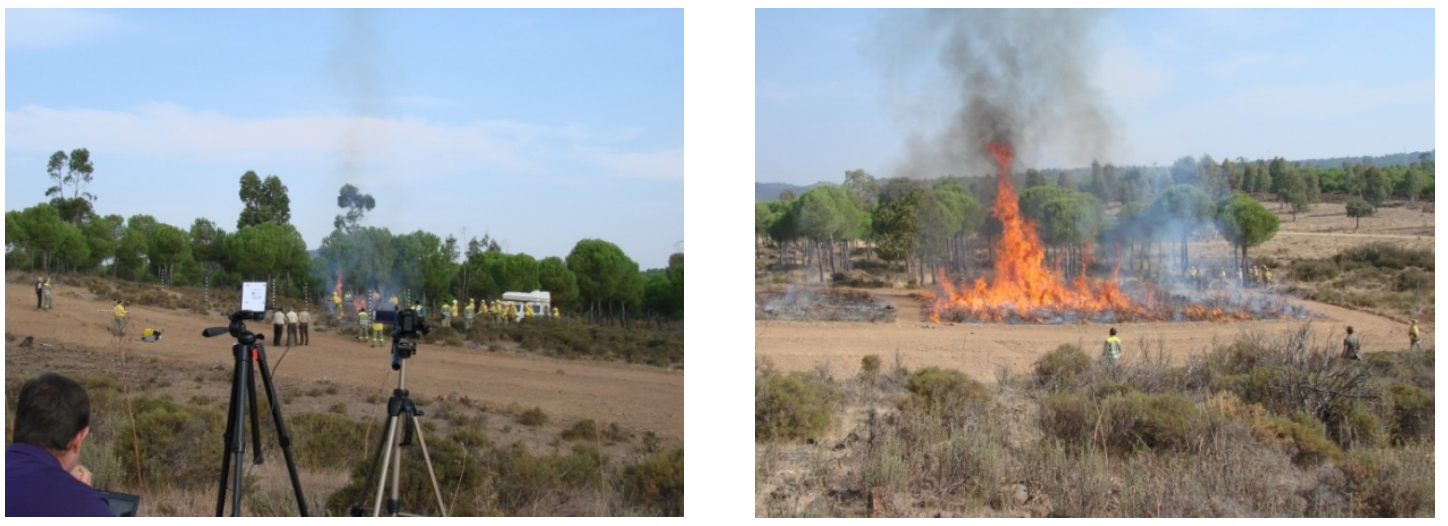

Fig. 14: Snapshot taken during the latest test carried out.

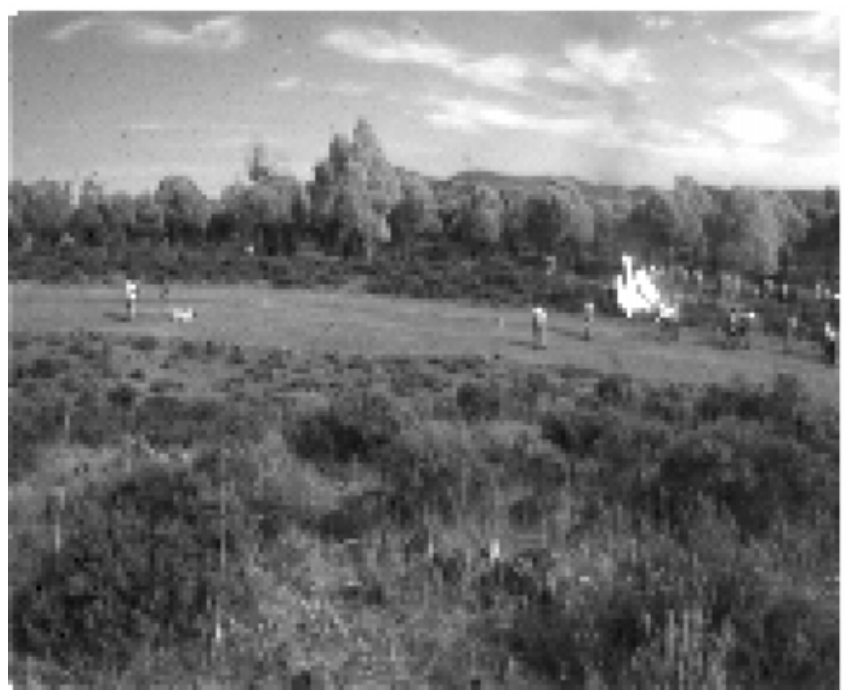

Fig. 15: Image sent via radio by $W i-F L I P$ for one of the alarms triggered. 
1 These results are promising, but we still think that several aspects of our prototype node must be

2 improved. The current lifetime of the system is competitive, taking into account that it can be

3 powered by small commercial batteries for around 10 hours. However, the objective is to have

4 the nodes running autonomously for at least several weeks. Energy harvesting is therefore

5 absolutely mandatory, even if the power consumption of the system is significantly reduced. We

6 have also noticed that the parameters of the algorithm still require further tuning. Specifically,

7 earlier detection could be probably attained with a longer time interval $T_{D_{M A X}}$. Its current value

8 forced the algorithm to dismiss spatio-temporal dynamics which corresponded to the beginning

9 of the fire, thus delaying the alarm trigger. The performance of Wi-FLIP has not yet been

10 analyzed in a real deployment. In such conditions, each node must deal with different kinds of

11 information coming from the network - the updating of routing tables, radio beacons, alarms

12 etc. - while keeping the algorithm working correctly. This is not a trivial issue and must be

13 adequately addressed. Finally, we estimate that at least four sensors per $\mathrm{km}^{2}$ will be necessary for adequate coverage, although this figure will heavily depend on the characteristics of the area to be surveyed. Nevertheless, it is important to reemphasize that vision-enabled wireless sensor networks are intended to enable low-cost fine-grained surveillance of locations with a high fire danger index. They are not, in principle, intended to cover very extensive areas with lots of sensors. Cost estimates for a system with the proposed areal coverage are really difficult to provide for the time being and depend on many parameters which would have to be taken into careful consideration before the system could be marketed: smart imager integration technology, the number of samples manufactured, the microprocessor chosen to run the system, the transceiver used etc. In any case, the scalable nature of the proposed system enables progressive implementation without incurring the huge costs of current automatic ground systems. 


\section{Conclusions}

2 The incorporation of vision into wireless sensor network nodes represents a remarkable step

3 forward in the possibilities of these networks in terms of forest fire detection. In this paper, a

4 new framework is defined by merging ground detection systems and WSN-based monitoring

5 systems in order to achieve early detection with greater robustness and reliability. It is based on

6 the design of a reliable vision algorithm and the ad-hoc implementation of a low-power smart

7 imager. These two key elements have been integrated into a prototype vision-enabled WSN

8 node. The results of the field tests carried out have demonstrated that the proposed approach

9 constitutes a sound basis for the future development of fine-grained spatio-temporal sensing

10 grids. Such future development should take into account numerous aspects not yet studied, for example how the performance of the vision algorithm varies for sensor-plume distances outside the range considered. Specifically, assuming that at least four sensors per $\mathrm{km}^{2}$ will be necessary 13 for adequate coverage, future tests should check the whole detection process for greater 14 distances than those of the experiments performed in this paper. Other features to be also 15 explored are energy harvesting techniques, further tuning of the parameters or fine adjustment 16 of the number of sensors for adequate coverage.

\section{Acknowledgements}

18 The authors would like to express their deep gratitude to Mr. Manuel Larios de la Carrera 19 (deputy director) and Mr. Salvador Benítez Moscoso (director), from the INFOCA Operational 20 Center for the province of Seville, for arranging the burns. This work is funded by MICINN 21 (Spain) through project TEC2009-11812, co-funded by the European Regional Development 22 Fund, by the Office of Naval Research (USA), through grant N000141110312, by the Spanish 23 Ministry of Science and Innovation through Project IPT-2011-1625-430000 and by the Spanish 24 Centre for Industrial Technological Development, co-funded by the European Regional Development Fund, through Project IPC-20111009. 


\section{References}

3 Akyildiz IF, Su W, Sankarasubramaniam Y, Cayirci E (2002) A survey on sensor networks.

4 IEEE Communications Magazine 40(8), 102-114.

5 Akyildiz IF, Melodia T, Chowdhury KR (2007) A survey on wireless multimedia sensor 6 networks. Computer Networks 51(4), 921-960.

7 Arrue BC, Ollero A, Martínez de Dios JR (2000) An intelligent system for false alarm reduction 8 in infrared forest fire detection. IEEE Intelligent Systems, 15(3), 64-73.

9 Chaczko Z, Ahmad F (2005) Wireless sensor network based system for fire endangered areas. 10 In 'Third Int. Conf. on Information Technology and Applications', Sidney, Australia, 477-484.

11 Chen T, Yin Y, Huang S, Ye Y (2006) Smoke detection for early fire-alarming system based on 12 video processing. In 'IEEE Int. Conf. on Intelligent Information Hiding and Multimedia Signal 13 Processing', Pasadena (CA), USA, 427-430.

14 de Vries JS, Kemp RA (1994) Results with a multispectral autonomous wildfire detection 15 system. In 'Proc. SPIE, Infrared Technology XX', San Diego (CA), USA, 18-28.

16 den Breejen E, Breuers M, Cremer F, Kemp RA, Roos M, Schutte K, de Vries JS (1998) 17 Autonomous Forest Fire Detection. In 'III International Conference on Forest Fire Research', 18 Coimbra, Portugal.

19 Doolin D, Sitar N (2005) Wireless sensors for wildfire monitoring. In 'SPIE Symposium on 20 Smart Structures and Materials', San Diego, USA, 477-484.

21 Eren G, Akan OB (2005) Multimedia communication in wireless sensor networks. Annales des 22 Telecommunications 60(7-8), 872-900. 
1 Fernández-Berni J, Carmona-Galán R, Carranza-González L (2008) A vision-based monitoring

2 system for very early automatic detection of forest fires. In 'First Int. Conf. on Modelling,

3 Monitoring and Management of Forest Fires', Toledo (Spain), 161-170.

4 Fernández-Berni J, Carmona-Galán R, Carranza-González L (2009) A VLSI-oriented and 5 power-efficient approach for dynamic texture recognition applied to smoke detection. In 'Int.

6 Conf. on Computer Vision Theory and Applications', Lisbon (Portugal), 307-314.

7 Fernández-Berni J, Carmona-Galán R, Carranza-González L, Cano-Rojas A, Martínez-Carmona

8 JF, Rodríguez-Vázquez A, Morillas-Castillo S (2010) On-site forest fire smoke detection by

9 low-power autonomous vision sensor. In 'VI International Conference on Forest Fire Research',

10 Coimbra, Portugal.

11 Fernández-Berni J, Carmona-Galán R, Carranza-González L (2011-1). A QCIF resolution focal12 plane array for low-power image processing. IEEE Journal of Solid-State Circuits 46(3), 66913680.

14 Fernández-Berni J, Carmona-Galán R, Liñán-Cembrano G, Zarándy A, Rodríguez-Vázquez A 15 (2011-2) Wi-FLIP: A wireless smart camera based on a focal-plane low-power image processor. 16 In 'Fifth ACM/IEEE Int. Conf. on Distributed Smart Cameras', Ghent (Belgium).

17 Fierens PI (2009) Number of sensors versus time to detection in wildfires. International Journal of Wildland Fire 18, 825-829.

Fire Watch (2004). Fire Watch: a commercial forest fire detection system. http://www.firewatch.de.

21 Gómez-Rodríguez F, Pascual-Peña S, Arrue BC, Ollero A (2002) Smoke detection using image processing. In 'IV International Conference on Forest Fire Research', Coimbra, Portugal.

Hefeeda M (2007) Forest fire modelling and early detection using wireless sensor networks. Technical report, School of Computing Science, Simon Fraser University. 
1 INSA (2000) FUEGO instrument design, prototype, construction and validation. Technical

2 report, INSA Ingeniería y Servicios Aeroespaciales.

3 Jacquemoud S, Verdebout J, Schmuck G, Andreoli G, Hosgood B, Hornig SE (1994)

4 Investigation of Leaf Biochemistry by Statistics. In 'Geoscience and Remote Sensing

5 Symposium', vol. 2 1239-1241.

6 Kremens R, Faulring J, Gallagher A, Seema A, Vodacek A (2003) Autonomous field7 deployable wildland fire sensors. International Journal of Wildland Fire 12, 237-244.

8 Krstinic D, Stipanicev D, Jakovcevic T (2009) Histogram-based smoke segmentation in forest

$9 \quad$ fire detection system. Information Technology and Control 38(3) 237-244.

10 Kuhrt E, Knollenberg J, Mertens V (2001) An automatic early warning system for forest fires.

11 Annals of Burns and Fire Disasters XIV(3), 151-155.

12 Machado R, Ribeiro G, Ditmore A, Romano M, Meireles W (2010) Online Paradise: A wireless 13 sensor network applied in the prevention and detection of forest fires. In 'VI International 14 Conference on Forest Fire Research', Coimbra, Portugal.

15 Pottie, G., Kaiser, W. (2000) Wireless integrated network sensors. Communication of the ACM $16 \quad 43(5), 51-58$.

17 Rothermel RC (1972) A mathematical model for predicting fire spread in wildland fuels. 18 Research paper, Intermountain Forest and Range Experiment Station, USDA Forest Fire 19 Service.

20 Schroeder D (2004) Evaluation of three wildfire smoke detection systems. Advantage Report, 21 FERIC Forest Engineering Research Institute of Canada, 5(24).

22 Schroeder D (2005) Operational Trial of the ForestWatch Wild_re Smoke Detection System. 23 Advantage Report, FERIC Forest Engineering Research Institute of Canada, 6(17). 
1 Sistema Bosque (1999) Sistema Bosque, Andalusian Forest Fire Suppression and Prevention

2 Service. http://waste.ideal.es/sistemabosque.htm.

3 Son B, Her Y, Kim J (2006) A design and implementation of forest-fires surveillance system

4 based on wireless sensor networks for South Korea mountains. International Journal of

5 Computer Science and Network Security 6(9), 124-130.

6 Stipanicev D, Stula M, Krstinic D, Seric L, Jakovcevic T, Bugaric M (2010) Advanced

7 automatic wildfire surveillance and monitoring network. In 'VI International Conference on

8 Forest Fire Research', Coimbra, Portugal.

9 Toreyin U, Dedeoglu Y, Enis-Cetin A (2007) Computer vision-based forest fire detection and

10 monitoring system. In 'International Wildland Fire Conference', Seville, Spain

11 Vicente J, Guillemant P (2002) An image processing technique for automatically detecting

12 forest fires. International Journal of Thermal Sciences 41(12), 1113-1120. 